\title{
Improve consensus via decentralized predictive mechanisms
}

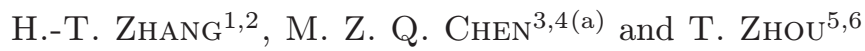 \\ ${ }^{1}$ Key Laboratory of Image Processing and Intelligent Control, Department of Control Science and Engineering, \\ Huazhong University of Science and Technology - Wuhan 430074, PRC \\ ${ }^{2}$ State Key Laboratory of Digital Manufacturing Equipment and Technology, Huazhong University of Science \\ and Technology - Wuhan, 430074, PRC \\ ${ }^{3}$ Department of Automation, Nanjing University of Science and Technology - Nanjing 210094, PRC \\ ${ }^{4}$ Department of Engineering, University of Leicester - Leicester LE1 7RH, UK, EU \\ ${ }^{5}$ Department of Modern Physics, University of Science and Technology of China - Hefei 230026, PRC \\ ${ }^{6}$ Department of Physics, University of Fribourg, Chemin du Muse - CH-1700 Fribourg, Switzerland
}

PACS 05.65.+b - Self-organized systems

PACS 87.17.Jj - Cell locomotion, chemotaxis

PACS 89.75.-k - Complex systems

\begin{abstract}
For biogroups and groups of self-driven agents, making decisions often depends on interactions among group members. In this paper, we seek to understand the fundamental predictive mechanisms used by group members in order to perform such coordinated behaviors. In particular, we show that the future dynamics of each node in the network can be predicted solely using local information provided by its neighbors. Using this predicted future dynamics information, we propose a decentralized predictive consensus protocol, which yields drastic improvements in terms of both consensus speed and internal communication cost. In natural science, this study provides an evidence for the idea that some decentralized predictive mechanisms may exist in widely-spread biological swarms/flocks. From the industrial point of view, incorporation of a decentralized predictive mechanism allows for not only a significant increase in the speed of convergence towards consensus but also a reduction in the communication energy required to achieve a predefined consensus performance.
\end{abstract}

Introduction. - Nature has presented numerous examples of collective behaviors in groups of animals, bacteria, cells and molecular motors. Such behaviors exist to permit sophisticated group functionalities such as foraging for food and defense against predators, which cannot be achieved by individual members. In this research field, one of the most general and attractive topics is the consensus problem [1-3], where groups of agents agree upon certain quantities such as position and temperature.

The most fascinating issue of biological flocks/swarms is the emergence of coordinated motion through decentralized or localized interactions without group leaders or the global view of the group, e.g. [4-6]. The interactions among neighbors can be simple (attraction/repulsion [7] or neighborhood alignment models [8]) or more complex (combinations of simple interactions like Couzin's model [9]) and can occur between neighbors in space or in

(a)E-mail: michael.chen@cantab.net; mzqchen@gmail.com an underlying network. Such interactions yield appealing ordering phenomena, and the collective dynamical systems arise in biological networks at multiple levels of abstraction, from interactions among molecules and cells [10] to the behavioral ecology of animal groups [4].

Most of the previous works analyzed performance improvements, such as increasing the consensus speed, making the formation more rigid and cohesive and improving the robustness to node/edge failures or to time delays, solely based on the information flow available at a given instant, e.g. [3,11-17]. Moreover, the consensus method has also been systematically investigated for both synchronous and asynchronous cases without employing any predictive mechanism [18]. Two important questions are thus naturally motivated: i) Are there any localinformation-based predictive intelligence embedded in flocking/swarming dynamics? ii) What is the role of such decentralized prediction mechanisms in consensus?

To answer the first question, one can refer to some experimental evidences e.g. [19-23], which strongly support the 
conjecture of the existence of some predictive mechanisms inside abundant biogroups. To answer the second question, as the preliminary work, we showed in our previous paper [24] that centralized predictive mechanism can yield ultrafast consensus without changing the communication topology. To further our investigation, we demonstrate that, by introducing some decentralized predictive mechanisms, it is possible to either significantly enhance the consensus speed obtainable with a fixed communication energy or to decrease the communication energy required to ensure a prescribed consensus speed.

In natural biogroups, recording past states (position, velocity, etc.) of one's neighbors, an agent can estimate their future states several steps ahead and then align to its neighbors' future states. This observation allows us to infer that individuals may use local information for decisionmaking, such as that used by bees to adapt to uncertain surroundings. In engineering applications, each agent typically has limited power to send messages, and thus a low sampling frequency is desirable. Since the predictive mechanisms can broadly expand the range of feasible sampling periods, which we will show later, such predictive protocols can be fairly useful for industrial applications. This paper aims to reveal the role of decentralized predictive mechanisms in coordinated behaviors.

Prediction based on local information. - In this section, we will show that each individual can predict the dynamics of its own merely using its local observation on its neighbors, provided that its memory length is long enough. In other words, "historical local information is equivalent to current global information". This observation is important since it may be the foundation stone of decentralized prediction capability embedded in biogroups.

We represent a network of interacting agents by a digraph $G=(\mathcal{V}, \mathcal{E}, \mathcal{A})$, where $\mathcal{V}=\left\{v_{1}, \ldots, v_{N}\right\}$ is the set of $N$ nodes, $\mathcal{E} \subset \mathcal{V} \times \mathcal{V}$ is the set of edges, and $\mathcal{A}$ is the adjacency matrix with entries $a_{i j} \geqslant 0$ denoting the edge weight of the interconnection from node $i$ to $j$. No selfcycle is allowed, hence $a_{i i}=0$ for all $i$. Let $x_{i}(k)$ denote the state of node $i$ at discrete-time instant $k$, representing any physical quantity. The nodes of a network have reached consensus if and only if $x_{i}=x_{j}$ for all $i$ and $j$. When the nodes are all in agreement, their common value is called the group decision value. If this value is $\bar{x}(0)=$ $1 / N\left(\sum_{i=1}^{N} x_{i}(0)\right)$, the network has reached the averageconsensus.

Single-integrator dynamic networks. We first consider the simplest non-predictive consensus protocol, i.e. the routine average-consensus protocol [3], for single-integrator networks. In the routine consensus protocol, the continuous-time dynamics of each individual $i$ is given by $\dot{x}_{i}(t)=u_{i}(t)$ with the consensus control signal $u_{i}(t)=\sum_{j=1}^{N} a_{i j}\left(x_{j}(t)-x_{i}(t)\right)$, then the whole system can be expressed by $\dot{x}(t)=L x(t)$ where $L=\left\{l_{i j}\right\}=\operatorname{diag}\left(\mathcal{A} \mathbf{1}_{N \times 1}\right)-\mathcal{A}$ represents the Laplacian matrix associated with the digraph $G$. If $x$ is the position vector of the group, this protocol just yields the consensus of individual positions, and has not considered the consensus of higher-order differentials of $x, e . g$. velocities and accelerations.

After discretization, the dynamics of each individual is denoted by $x_{i}(k+1)=x_{i}(k)+\epsilon u_{i}(k)$ and the general behavior of the whole network is represented by

$$
x(k+1)=P_{\epsilon} x(k),
$$

where $P_{\epsilon}=I_{N}-\epsilon L$ is the state matrix of the considered network, $\epsilon$ denotes the sampling period or step size associated with the discrete-time process, and $I_{N}$ is an $N$-dimensional identity matrix. Let $d_{\max }=\max _{i}\left(l_{i i}\right)$ denote the maximum node out-degree of $G$. For a balanced network with at least one spanning tree therein, if the sampling period $\epsilon \in\left(0,1 / d_{\max }\right)$, then the above routine consensus protocol ensures global asymptotic convergence to consensus [3]. In the rest of the paper, for brevity, we denote average-consensus by consensus.

Using the network dynamics (1), the $m$-step-ahead future dynamics of any individual $i$ is given by

$$
x_{i}(k+m)=e_{i} P_{\epsilon}^{m} x(k)
$$

with the row extracting operator $e_{i}=\left[0, \ldots, 0,1_{i \text {-th }}\right.$, $0, \ldots, 0]_{1 \times N}$. Naturally, it seems that the global information including the state matrix $P_{\epsilon}$ and the current state $x(k)$ are needed to predict the future states of the $i$-th individual. We, however, will show that, even if such global information is unavailable to each node (which is typical in biogroups and engineering multi-agent systems), each individual can still estimate its own and neighbors' future states by using the present and past information it collects from its neighbors.

Let $Z_{i}(k)=\left[z_{1, i}(k), \cdots, z_{N, i}(k)\right]^{T}$ denote the historical state sequence of length $N$ for the $i$-th individual with $z_{l, i}(k)=x_{i}(k+1-l), l=1, \cdots, N$, one has that

$$
Z_{i}(k)=\Phi_{i} x(k-N),
$$

and the future dynamics of individual $i$ and its neighbors $j$ can be iterated as follows:

$$
x_{i}(k+m)=e_{1} A_{i}^{m} Z_{i}(k), x_{j}(k+m)=B_{j, i} A_{i}^{m-1} Z_{i}(k)
$$

with $\Phi_{i}=\left[\left(e_{i} P_{e}^{N}\right)^{T}, \cdots,\left(e_{i} P_{e}\right)^{T}\right]^{T}$ and

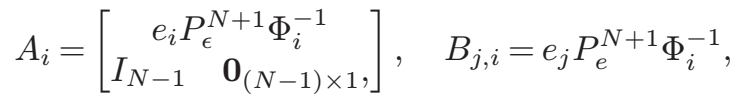

provided that $\Phi_{i}$ is invertible. Here, individual $j$ is a neighbor of individual $i, m=1, \cdots, H_{p}$ and $H_{p}$ is future steps to be predicted, namely the prediction horizon.

Therefore, for any individual $i$, provided that the parameters $A_{i}$ and $B_{j, i}$ in eq. (5) can be appropriately estimated, the future dynamics of its neighbors can be effectively predicted solely using the historical local state sequences $Z_{i}(k)$ and $Z_{j}(k)$ observed by individual $i$. 
Comparing eq. (4) with eq. (2), we note that "current global information" including $P_{\epsilon}$ and $x(k)$ is no longer required for state prediction as this information can be recovered from "historical local observations" once the local correlation information $A_{i}$ and $B_{i, j}$ have been satisfactorily estimated. In this sense, "historical local information is equivalent to current global information".

Estimate of $A_{i}$ and $B_{j, i}$ can be realized provided that each individual possesses a memory of sufficient capacity, allowing it to store the length- $N$ historical state sequences of $x_{i}(k)$ and $x_{j}(k)$ ( $j$ is a neighbor of $i$ ). Here, $N_{p}$ is the diameter of the graph $G$, i.e. the longest pathway length between each node-pair of $G$. Therefore, by

$$
x_{j}(k-n)=\alpha_{j, i} Z_{i}(k-n-1), \quad n=0,1, \cdots, N-1,
$$

one can use least-square estimation (LSE) [25] to obtain the estimates $\hat{\alpha}_{j, i}$ of the row vectors $\alpha_{j, i}$. These estimates $\hat{\alpha}_{j, i}$ are then used to reproduce the matrix $e_{j} P_{e}^{N+1} \Phi_{i}^{-1}$ in $A_{i}$ and $B_{i, j}$ (see eq. (5)).

Furthermore, in natural swarms/flocks, individuals can escape/enter the neighborhood of an individual, making the network topology switch continuously. Fortunately, if the topology modification rate is low enough, the proposed decentralized prediction remains feasible. Since the prediction is based on the historical information of the last $2 N$ steps before current time $k$ (see eq. (6)), and is aimed at the prediction of the future $H_{p}$ steps (see eq. (4)), the decentralized prediction protocol (6) remains valid provided that the topology remains constant for $2 N+H_{p}$ steps. Thus, the topology changing frequency cannot be higher than $\frac{1}{\left(2 N+H_{p}\right) \epsilon}$. Hereby, the relationship between any agent $i$ and its neighbor $j$ remains identical as shown in eq. (4) during the recent $2 N+H_{p}$ steps, and hence using the LSE method in eq. (6) can yield unbiased estimate and minimum-variance unbiased estimate of $\alpha_{i j}$ for the noisefree and white-noise cases, respectively [25]. In practical applications, if the topology of the network changes quite frequently, the sampling period $\epsilon$ can be reduced, i.e., the interaction frequency of each neighboring pair can be increased, to guarantee the upper bound of topology changing frequency.

The decentralized predictive protocol (6) is illustrated on the 5-node network shown in fig. 1, in which the topology is changed from fig. 1(a) to fig. 1(b) at the 50th step. Simulations shows that, before the topology switch, the prediction error $\delta_{p}(k)$ remains small (less than $10^{-9}$ ). Bearing in mind the prediction rule (4) and the identification rule (6), the prediction error of node $i$ is defined as $\delta_{p, i}(k+m)=\left\|x_{i}(k+m)-\hat{x}_{i}(k+m)\right\|_{2}$, with predicted state $\hat{x}_{i}(k+m)=e_{i} \hat{A}_{i}^{m} Z_{i}(k)$ and $\hat{A}_{i}=\left[\begin{array}{c}\hat{\alpha}_{i, i} \\ I_{N-1} \mathbf{0}_{(N-1) \times 1}\end{array}\right]$. When the topology switch occurs at the 50th step, the prediction error $\delta_{p, i}(k)$ rises sharply to approach 0.01 and then begins to oscillate as a result of the transient adaptive process of the prediction. After less than $N$ steps, $\delta_{p, i}(k)$ settles down to a level lower than $10^{-10}$. Thus, both the prediction accuracy and the adaptive capability for topology variations are demonstrated.
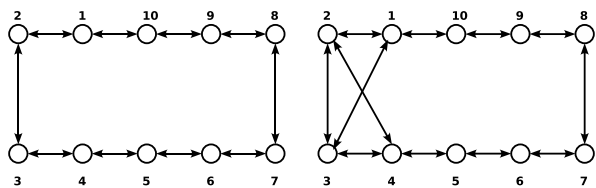

(a)

(b)

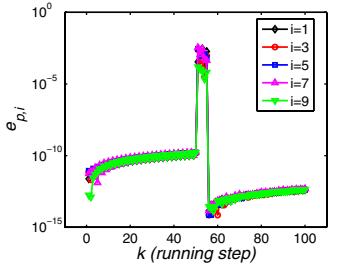

(c)

Fig. 1: (Color online) The network topology is switched from (a) to (b) at the 50th running step; (c) gives the prediction error $\delta_{p}(k)$ on a single-integrator network with switching topology. Here, $\epsilon=0.35, a_{i j}=1$ if $(i, j) \in \mathcal{E}$, otherwise $a_{i j}=0$, the initial state of each node is selected randomly in the range $[0,1]$, and each point is an average over 500 independent runs.

Note that: i) The tiny prediction error is due to the inevitable LSE identification error of $\hat{\alpha}_{j, i}$; ii) For prediction, each node is required to restore the global state from local observation. Thus, each node needs to store sufficiently long historical state sequence of its neighbors and itself. iii) We assume that the sampling and decisionmaking time of all the nodes are synchronized, which is a common assumption for many existing results in literature concerning discrete-time analysis of collective behaviors $[3,8,12,14,15,24]$. However, even for the asynchronous case, our method still works since the dynamical relationship $\alpha_{i j}$ extracted from neighboring interactions by eq. (6) is independent of the sampling instant but solely determined by the network's dynamics $\dot{x}=L x$.

Multi-integrator dynamic networks. The abovementioned decentralized prediction also holds for more general networks, say, multi-integrator networks. In the routine consensus protocol, the continuous-time dynamics of each individual $i$ is given by $x_{i}^{(n)}(t)=u_{i}(t)$ with the consensus control signal $u_{i}(t)=-\sum_{j=1}^{N} a_{i, j}\left[\left(x_{i}(t)-\right.\right.$ $\left.\left.x_{j}(t)\right)+\sum_{m=1}^{n-1} \gamma_{m} \Delta\left(x_{i}^{(m)}(t)-x_{j}^{(m)}(t)\right)\right]$ where the superscript $(n)$ denotes the $n$-order differential, and $\gamma_{m}$ is the coupling strength between the $m$-order state derivatives.

Therefore, the whole system can be expressed by $\dot{\xi}(t)=$ $\Gamma \xi(t)$ with

$$
\Gamma=\left[\begin{array}{ccccc}
\mathbf{0}_{N} & I_{N} & \mathbf{0}_{N} & \cdots & \mathbf{0}_{N} \\
\mathbf{0}_{N} & \mathbf{0}_{N} & I_{N} & \cdots & \mathbf{0}_{N} \\
\vdots & \vdots & & \ddots & \vdots \\
\mathbf{0}_{N} & \cdots & \mathbf{0}_{N} & \cdots & I_{N} \\
-L & -\gamma_{1} L & -\gamma_{2} L & \cdots & -\gamma_{n-1} L
\end{array}\right]
$$

and

$$
\begin{aligned}
\xi(t)= & {\left[x_{1}(t), \cdots, x_{N}(t), \dot{x}_{1}(t), \cdots, \dot{x}_{N}(t), \cdots, x_{1}^{(n)}(t),\right.} \\
& \left.\cdots, x_{N}^{(n)}(t)\right]^{T} .
\end{aligned}
$$




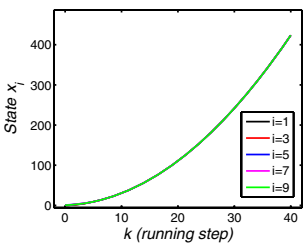

(a)

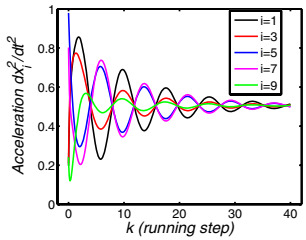

(c)

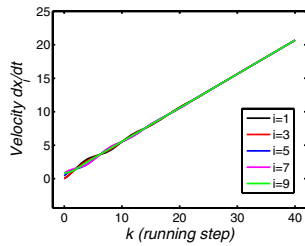

(b)

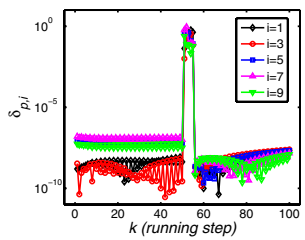

(d)
Fig. 2: (Color online) Consensus procedures of (a) state $x$, (b) velocity $\dot{x}$ and (c) acceleration $\ddot{x}$; (d) gives the prediction error $\delta_{p}(k)$ on a triple-integrator network with switching topology from fig. 1(a) to (b) at the 50th running step. Here, $\epsilon=0.30, \gamma_{1}=\gamma_{2}=2, a_{i j}=1$ if $(i, j) \in \mathcal{E}$, otherwise $a_{i j}=0$, the initial state of each node is selected randomly in the range $[0,1]$, and each point is an average over 500 independent runs.

In comparison with the single-integrator networks in the previous subsection, the virtue of this protocol lies in yielding the consensus of not only positions $x$ but also velocities $\dot{x}$, accelerations $\ddot{x}$, etc.

Analogous to the previous subsection, after discretization over $\dot{\xi}(t)=\Gamma \xi(t)$, one can establish the relationship between the historical state sequence $Z_{i}(k)$ of agent $i$ and its neighbors $j \in \mathcal{N}(i) \cup i$, and thereby design the corresponding decentralized predictive protocol for multiintegrator networks. We demonstrate its prediction performances on a triple-integrator dynamic network $(n=3)$ in fig. 2, where the topology changes from fig. 1(a) to fig. 1 (b) at the 50th step. Simulations show that, apart from the oscillations shortly after the switching time, the prediction error $\delta_{p}(k)$ remains small $\left(<10^{-9}\right)$. Note that the larger prediction errors and switching oscillations of fig. 2 in comparison with fig. 1(c) root in the much more complex dynamics of the triple-integrator network.

Decentralized predictive protocol. - We now introduce a predictive consensus protocol to improve the consensus performance. We only present the case of single-integrator network, and the counterpart of multiintegrator network can be easily derived. We consider the routine dynamics given in 4 with one-step prediction and replace them by the following model predictive control (MPC) protocol:

$$
x_{i}(k+1)=e_{1} A_{i} Z_{i}(k)+v_{i}(k)
$$

where $v_{i}(k)$ is an additional term representing the predictive mechanism to be determined. Using (7), the future states of agent $i$ and its neighbors $j$ can be predicted based on the available historical state sequence $Z_{i}(k)$ as follows:

$$
X_{i}(k+1)=P_{Z_{i}} Z_{i}(k)+P_{U_{i}} U_{i}(k),
$$

$$
X_{j}(k+1)=P_{Z_{j}} Z_{i}(k)+P_{U_{j}} U_{i}(k),
$$

where $\quad X_{i}(k+1)=\left[x_{i}(k+1), \cdots, x_{i}\left(k+H_{p}\right)\right]^{T}, \quad U_{i}(k)=$ $\left[v_{i}(k), \cdots, v_{i}\left(k+H_{p}-1\right)\right]^{T}$ and

$$
\begin{aligned}
& P_{Z_{i}}=\left[\left(e_{1} A_{i}\right)^{T}, \cdots,\left(e_{1} A_{i}^{H_{p}}\right)^{T}\right]_{H_{p} \times N}^{T}, \\
& P_{Z_{j}}=\left[B_{j, i}^{T},\left(B_{j, i} A_{i}\right)^{T}, \cdots,\left(B_{j, i} A_{i}^{H_{p}-1}\right)^{T}\right]_{H_{p} \times N}^{T},
\end{aligned}
$$

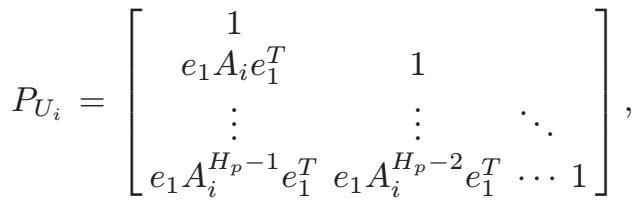

$$
\begin{aligned}
& P_{U_{j}}=\left[\begin{array}{cccc}
0 & & & \\
B_{j, i} e_{1}^{T} & 0 & & \\
\vdots & \vdots & \ddots & \\
B_{j, i} A_{i}^{H_{p}-2} e_{1}^{T} & B_{j, i} A_{i}^{H_{p}-3} e_{1}^{T} & \cdots & 0
\end{array}\right] \text {. }
\end{aligned}
$$

Bearing in mind that $A_{i}$ and $B_{j i}$ in eq. (5) can be accurately approximated based on local observation, we ignore the identification error (i.e., $\hat{\alpha}_{j, i}=B_{j, i}$ ) in the rest of this paper.

The natural interpretation of the decentralized predictive control mechanism is as follows. Each individual observes and stores the historical trajectories of itself and its neighbors, predicts its neighbors' future states using, e.g., (8) and (9), and computes some control signal to minimize its predicted state difference with its neighbors.

We now represent the state difference between individual $i$ and its neighbors by a vector $\Delta \mathbb{X}_{i}(k+1)=$ $\operatorname{row}\left\{\Delta X_{r, s}(k+1) \mid r, s \in \mathcal{N}(i) \cup i \quad\right.$ and $\left.\quad r>s\right\} \quad$ where $\Delta X_{r, s}(k+1)=X_{r}(k+1)-X_{s}(k+1)$ and the notation $y=\operatorname{row}\left\{y_{i}\right\}$ represents that the $i$-th row of $y$ is $y_{i}$. It then follows from eqs. (8) and (9) that $\Delta \mathbb{X}_{i}(k+1)=P_{\mathbb{Z}_{i}} Z_{i}(k)+P_{\mathbb{U}_{i}} U_{i}(k)$ with $r, \quad s \in \mathcal{N}(i) \cup i$, $r>s, P_{\mathbb{Z}_{i}}=\operatorname{row}\left\{P_{Z_{r}}-P_{Z_{s}}\right\}$ and $P_{\mathbb{U}_{i}}=\operatorname{row}\left\{P_{U r}-P_{U s}\right\}$.

To solve the consensus problem, we first set the moving horizon optimization index that defines the decentralized MPC consensus problem as follows:

$$
J_{i}(k)=\left\|\Delta \mathbb{X}_{i}(k+1)\right\|_{Q}^{2}+\left\|U_{i}(k)\right\|_{R}^{2},
$$

where $Q$ and $R$ are compatible real, symmetric, positive definite weighting matrices, and $\|M\|_{Q}^{2}=M^{T} Q M$. In general, the weighting matrices can be set as $Q=$ $q I_{H_{p} N_{i}\left(N_{i}+1\right) / 2}(q>0)$ and $R=I_{H_{p}}$, where $N_{i}$ is the number of the neighbors of individual $i$. In the index (10), the first term penalizes the state difference between agent $i$ and each of its neighbor over the future $H_{p}$ steps, while the second term penalizes the additional MPC energy $U_{i}(k)$. To minimize $(10)$, we compute $\partial J_{i}(k) / \partial U_{i}(k)=0$, and obtain the optimal MPC action as

$$
v_{i}(k)=P_{M P C, i} Z_{i}(k),
$$




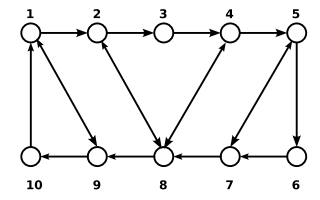

(a)

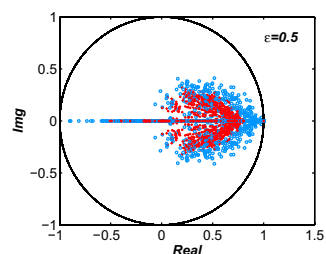

(b)

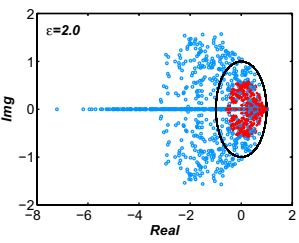

Fig. 3: (Color online) Eigenvalue distributions for different $\epsilon$. Blue (o) and red $(+)$ represent the cases of $P_{\epsilon}$ and $W$ (see eq. (12)) over 100 runs, respectively. The simulations are implemented on the balanced network with topology given in (a). The eigenvalue clusters of the MPC method are snapshot at the 50th running step. The black circle denotes the unit circle in the complex plane. Here, $H_{p}=7, q=0.015$, and each entry $l_{i j}(j \neq i$, and $(i, j) \in \mathcal{E})$ is chosen randomly in $[-1,0)$ such that the resulting network is balanced. It is calculated that the associated values of $d_{\max }$ lie in $[0.9,2.7]$.

with $\quad P_{M P C, i}=-[1,0, \cdots, 0]_{1 \times H_{p}} \cdot\left(P_{\mathbb{U}_{i}}^{T} Q P_{\mathbb{U}_{i}}+R\right)^{-1} P_{\mathbb{U}_{i}}^{T} \times$ $Q P_{\mathbb{Z}_{i}}$. Substituting eq. (11) into eq. (7) yields

$$
Z(k+1)=W Z(k)
$$

with $W=\operatorname{diag}\left\{A_{i}+e_{1}^{T} P_{M P C, i}\right\}_{i=1, \ldots, N} \quad$ and $\quad Z(k)=$ $\left[Z_{1}(k)^{T}, \ldots, Z_{N}(k)^{T}\right]^{T}$.

It can be shown that, for an $N$-node balanced network with dynamics determined by (12), provided that $\hat{\alpha}_{j, i}=B_{j, i}$, then the system state $x(k)$ asymptotically converges to the equilibrium point $\bar{x}(0) \boldsymbol{1}_{N}$ with $\bar{x}(0)=1 / N \sum_{i=1}^{N} x_{i}(0)$ if and only if assumptions $A 1$ and A2 hold:

A1: $\quad \rho\left(W-\mathbf{1}_{N^{2}} \boldsymbol{1}_{N^{2}}^{T} / N^{2}\right)<1$, where $\rho(\cdot)$ denotes the matrix spectral radius;

A2: $\boldsymbol{1}_{N}^{T} \cdot\left(A_{i}+e_{1}^{T} P_{M P C, i}\right)=\mathbf{1}_{N}^{T}(i=1, \ldots, N)$.

It can be seen from fig. 3 that $A 1$ is rational. More precisely, compared with the eigenvalue cluster of $P_{\epsilon}$, the one of $W$ (see eq. (12)) is always much smaller and closer to the origin except the single eigenvalue at 1 , which well explains the higher consensus speed of the present decentralized MPC protocol. More significantly, when $\epsilon$ is increased beyond $1 / d_{\max }$ (see fig. $3(\mathrm{~b})$ where $\epsilon=2$ ), some of the eigenvalues of $P_{\epsilon}$ start escaping the unit circle, making the disagreement function diverge, whereas the eigenvalue cluster of $W$ remains inside the unit circle, which ensures its convergence. In brief, the role of the matrix $P_{M P C}$ in eq. (12) is to drive back the escaping eigenvalues towards the origin, causing the improvement.

The condition A2 can be interpreted as that the internal prediction-related forces are counteracted. It can

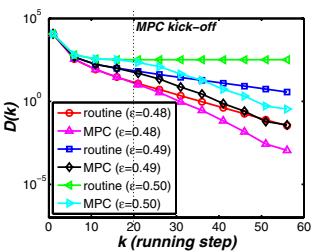

(a)

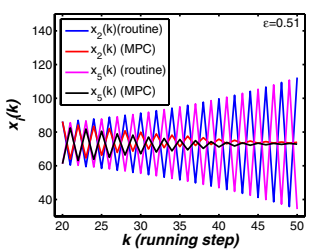

(c)

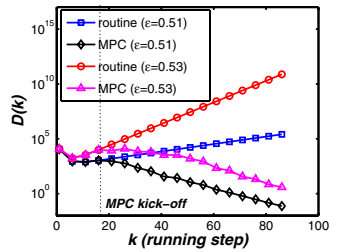

(b)

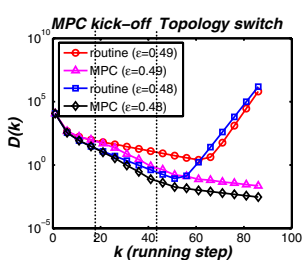

(d)
Fig. 4: (Color online) Consensus performance comparison of routine and predictive protocols on balanced network fig. 1(a). (a) $\epsilon \leqslant 1 / d_{\max }\left(d_{\max }=2\right.$, convergent case of the routine protocol); (b) $\epsilon>1 / d_{\max }$ (divergent case of the routine protocol); (c) state evolution for $\epsilon=0.51$; (d) consensus performance with switching topology. Here, the balanced network topology is switched from fig. 1(a) to fig. 1(b) at the 43rd running step. Here, $a_{i j}=1$ if $(i, j) \in \mathcal{E}$, otherwise $a_{i j}=0$, the initial state $x_{i}(0)$ is selected randomly in $[0,150], H_{p}=7, N=10$, $q=0.015$. Each point is an average over 500 independent runs.

be numerically checked that, in the 2-dimensional space spanned by the parameters $H_{p}$ and $q$, there is a fairly large region in which $A 2$ is fulfilled (such as the common parameter settings region $H_{p} \in[1,10]$ and $\left.q \in[0.01,10]\right)$.

To illustrate the advantages of the predictive consensus protocol, we present some simulation results comparing the convergence speeds using the routine protocol in eq. (1) and the proposed predictive protocol in eq. (12) on a 10-node network given in fig. 1(a). Since the objective is to reach average-consensus, the instantaneous disagreement index is typically set as $D(k)=\left\|x(k)-\mathbf{1}_{N} \bar{x}(0)\right\|_{2}^{2}$, for which smaller value implies more "consensus".

In the convergent case of the routine protocol [3], e.g. for $\epsilon \leqslant 1 / d_{\max }$, as shown in fig. 4(a), the decentralized MPC consensus protocol (12) yields an increase of the convergence speed towards average-consensus (by a factor of 3 approximately). Furthermore, even when the routine convergence conditions are violated, i.e. $\epsilon>1 / d_{\max }$, it is observed in figs. 4(b) and (c) that the decentralized MPC consensus protocol still allows asymptotic convergence to average-consensus. Thus, the range of sampling period $\epsilon$ leading to asymptotic convergence towards consensus can be expanded. Interestingly, one may notice the slight oscillations of MPC consensus protocol's $D(k)$ curve in figs. 4(a) and (b) which root in the online identification and adaptation processes performed by each node.

To further demonstrate the decentralized MPC's superiority in handling network topology switch, we compare the consensus performances of MPC and routine protocol in fig. 4(d), in which the topology is switched from fig. 1(a) to fig. $1(\mathrm{~b})$ at the $43 \mathrm{rd}$ running step. For the 
routine protocol, since $\{0.48,0.49\} \in[1 / 3,1 / 2](1 / 2$ and $1 / 3$ are the $1 / d_{\max }$ values of the topologies considered in fig. 1(a) and (b), respectively), the consensus is broken shortly after the topology switch. However, the consensus holds for the decentralized MPC protocol since it can adapt to the topology changes and use the additional MPC term (see eq. (7)) to steer each node towards its neighbors. Thus, its potential for industrial applications is quite clear. For example, in multi-robot formation control, each robot can memorize the historical dynamics of its neighbors and itself for prediction, and then gain quicker velocity consensus at a low cost of interactions.

One may notice that, in our control approach, each agent has been equipped with predictive capability, which could somewhat increase the controller cost. Fortunately, in our previous work $[15,24]$ and some pinning control methods [26], it has been demonstrated that, if just a few nodes know the future dynamics of the target, the collective behavior can still be effectively synchronized. Thus, this pinning control method may be used to further decrease the prediction cost in our future investigation.

Conclusions. - To extract the role of predictive mechanisms in natural biogroups with distributed interactions, we incorporated a decentralized predictive mechanism into each node of a network, and consequently designed a decentralized consensus scheme to mimic the predictive intelligence. We also illustrated the value of such predictive protocol for the analysis of emergent behaviors and for the design of autonomous consensus networks.

Within a centralized framework in our previous work [24], the future dynamics of each node can be easily derived provided that the current global state and topology of the whole network are available. However, practically, an individual usually lacks such global knowledge and can only observe its neighbors. Thus, we implemented some prediction mechanism on general multi-integrator networks and demonstrated that the future behavior of each individual and its neighbors can be accurately predicted by solely using a sufficiently long historical local information sequence observed by itself.

Based on the above effective decentralized prediction, we have incorporated an additional MPC term into the routine consensus protocol [3] to minimize the future state difference between each node and its neighbors. The comparison between the routine [3] and the proposed predictive consensus protocols led to two conclusions: i) each node's local information-based vision into the future can significantly accelerate the consensus speed of the whole group; ii) the feasible sampling period range is broadly expanded by this decentralized predictive mechanism.

Furthermore, to verify the generality of our conclusions, we have examined the capability of predictive mechanism in two popular flock/swarm models, the Vicsek model and the attractive/repulsive model [15]. The results also strongly imply that the decentralized predictive protocol has the capabilities of improving the synchronization performance while decreasing the communication cost.

For natural science, this work contribute to explaining why individuals of biological flocks/swarms do not communicate very frequently but just now and then during the whole dynamic process. For industrial applications, the consensus speed can be significantly enhanced while the communication energy or cost is reduced. All these merits are merely at the cost of allowing agents to make predictions solely based on local information sequence.

\section{REFERENCES}

[1] Grégoire G. and Chaté H., Phys. Rev. Lett., 92 (2004) 025702 .

[2] Aldana M., Dossetti V., Huepe C., Kenkre V. M. and Larralde H., Phys. Rev. Lett., 98 (2007) 095702.

[3] Olfati-Saber R. and Murray R., IEEE Trans. Autom. Control, 49 (2004) 1520.

[4] Okubo A., Adv. Biophys., 22 (1986) 1.

[5] Conradt L. and Roper T. J., Nature, 421 (2003) 155.

[6] Couzin I. D., Krause J., Franks N. R. and Levin S. A., Nature, 433 (2005) 513.

[7] Gazi V. and Passino K. M., IEEE Trans. Autom. Control, 48 (2003) 692.

[8] Vicsek T., Czirók A., Ben-Jacob E., Cohen I. and Shochet O., Phys. Rev. Lett., 75 (1995) 1226.

[9] Couzin I. D., Krausea J., James R., Ruxton G. D. and Franks N. R., Theor. Biol., 218 (2002) 1.

[10] Miller M. B. and Bassler B. L., Annu. Rev. Microbiol., 55 (2001) 165.

[11] Fiedler M., Czech. Math. J., 23 (1973) 298.

[12] Olfati-Saber R., Proc. Am. Control Conf., 4 (2005) 2371.

[13] Yang W., Cao L., Wang X. F. and Li X., Phys. Rev. E, 74 (2006) 037101.

[14] Li W., Zhang H. T., Chen M. Z. Q. and Zhou T., Phys. Rev. E, 77 (2008) 021902.

[15] Zhang H. T., Chen M. Z. Q. and Zhou T., Phys. Rev. E, 79 (2009) 016113.

[16] Zhang J., ZhaO Y., Tian B., Peng L. Q., Zhang H. T., Wang B. H. and Zhou T., Physica A, 388 (2009) 1237.

[17] Liu Y. and Passino K. M., IEEE Trans. Autom. Control, 51 (2006) 1734.

[18] Bertsekas D. P. and Tsitsiklis J. N., Parallel and Distributed Computation: Numerical Methods (Althena Scientific, Belmont, Mass.) 1997.

[19] Woods E. F., Nature, 184 (1959) 842.

[20] Montague P. R., Dayan P., Person C. and Sejnowski T. J., Nature, 377 (1995) 725.

[21] Gottfried J. A., Doherty J. O. and Dolan R. J., Science, 301 (2003) 1104.

[22] Summerfield C., Egner T., Greene M., Koechlin E., Mangles J. and Hirsch J., Science, $\mathbf{3 1 4}$ (2006) 1311.

[23] Melcher D., Nat. Neurosci., 10 (2007) 903.

[24] Zhang H. T., Chen M. Z. Q., Zhou T. and Stan G. B., $E P L, 83$ (2008) 40003.

[25] LJung L., System Identification: Theory for the User (Prentice-Hall, Inc., Englewood Cliffs, NJ) 1999.

[26] Zhou J., Lü J. and Lu J., Automatica, 44 (2008) 996. 